\title{
Let there be oxygen and T cells
}

\author{
Paul R. Walker
}

Centre for Translational Research in Onco-Hematology, Department of Internal Medicine Specialties, University of Geneva and Division of Oncology, Geneva University Hospitals, Geneva, Switzerland.

\begin{abstract}
The stroma of solid tumors can exclude or limit immune infiltration, or lead to the recruitment of tumor-promoting rather than tumor-attacking immune cells. This finding was reported by Jayaprakash et al. in this issue of the $J C l$, and it was particularly prominent in the hypoxic zones of tumors in the transgenic adenocarcinoma of the mouse prostate (TRAMP) cancer models. A current clinical goal of immune checkpoint blockade (ICB) is to extend its utility to more patients by converting immunologically "cold" tumors that do not provoke a strong immunological response to "hot" tumors that are invaded by swarms of T cells. When the underlying cause is hypoxia linked, the therapeutic combination of simultaneous targeting of hypoxia and immune checkpoints merits exploration in future clinical trials.
\end{abstract}

\section{Targeting hypoxia as a rational treatment approach to combine with immunotherapy} Immune checkpoint inhibitors are considered a cancer immunotherapy success story; nevertheless, only a minority of patients treated with immune checkpoint blockade (ICB) show clinical benefit (1). Intensive research efforts have revealed several potential biomarkers for ICB responsiveness, and new combination treatment regimens are being proposed to improve the response rate $(1,2)$. However, which combinations should be prioritized for clinical testing? Arguably, if there are common features of malignant tumors that act upstream of several ICB resistance factors, these should be high on the list. A prime candidate is hypoxia, which has long been considered a negative prognostic indicator for many solid tumors, associated with poor response to chemotherapy and radiotherapy, and linked to tumor progression through mechanisms such as promotion of stemness, cancer cell survival, invasion, angiogenesis, and metastasis (3). More recently, hypoxia has been correlated with an immunosuppressive tumor microenvironment (TME) (4), with well-described effects on the CD ${ }^{+}$ $\mathrm{T}$ cells that are proposed to be the primary effector cells elicited by ICB (5).

What approaches can be envisaged to manipulate hypoxia and thereby test its impact on cancer progression in the context of immunotherapy? Broadly, the choice can be between minimizing hypoxia itself or blocking its consequences. The bestdescribed cellular regulator of the hypoxia response is the hypoxia-inducible factor (HIF) pathway (6). HIF- $\alpha$ subunits are stabilized under low oxygen levels and pair with a constitutively expressed $\beta$-chain, leading to nuclear translocation and transcription of genes with a hypoxia-responsive element in their promotor. Compounds targeting HIFs are actively investigated as cancer therapeutics (3), but caution is warranted when antitumor immunity is the objective. Indeed, immune cells (including $\mathrm{T}$ cells and myeloid cells) can stabilize HIF- $1 \alpha$ in response to various stimuli in an oxygen-independent manner (7), and HIF inhibition or absence can impair immune effector functions. A more direct way of altering tumor oxygenation is to promote respiratory hyperoxia though supplemental oxygen delivery, as recently tested in mouse lung tumor models (8). This result-

Related Article: p. 5137

ed in decreased tumor hypoxia, weakened immunosuppression in the TME, and prolonged survival when mice were cotreated with immunotherapy. Although this approach demonstrated the importance of oxygen availability in cancer therapy, routine clinical translation is expected to be challenging. Hypoxia reduction can also be achieved by inhibiting oxygen consumption of tumors using the metabolic drug metformin, suggested to impede cancer progression and to promote responsiveness to immunotherapy (9). Nevertheless, the mechanisms of action of metformin are multiple, and enhancement of antitumor immunity can also occur through hypoxiaindependent mechanisms (10). Another view of hypoxia is that it represents a relatively tumor-specific marker suitable for targeting of cytotoxic drugs, thereby limiting systemic toxicity. This vision led to the development of hypoxia-activated prodrugs (HAPs), which require enzymatic reduction to release a cytotoxic effector molecule able to kill dividing tumor cells, a process only occurring under low oxygen tensions (11). There are certain caveats to this simplistic mode of action that might limit in vivo efficacy and tumor selectivity. First, limited oxygen availability is a feature of many healthy tissues, particularly secondary lymphoid tissues in which there are also dividing cells and oxygen levels of approximately $2.5 \%$ (12). However, although earlier-developed HAPs required only mild hypoxia for their activation (class I compounds), class II compounds are now available that require more severe hypoxia (11), thereby improving tumor selectivity $(3,11)$. Second, cancer cells in the hypoxic regions (i.e., the cells most efficiently targeted by HAPs) may have undergone adaptations promoting chemotherapy resistance, for example, through apoptosis resistance, modification of DNA repair mechanisms, or overexpression of ABC transporters (3). Despite the conceptual interest in HAPs, the results of clinical trials have been somewhat disappointing to date (11), which prompts interest in combining HAPs with other therapies, including immunotherapy. 
A

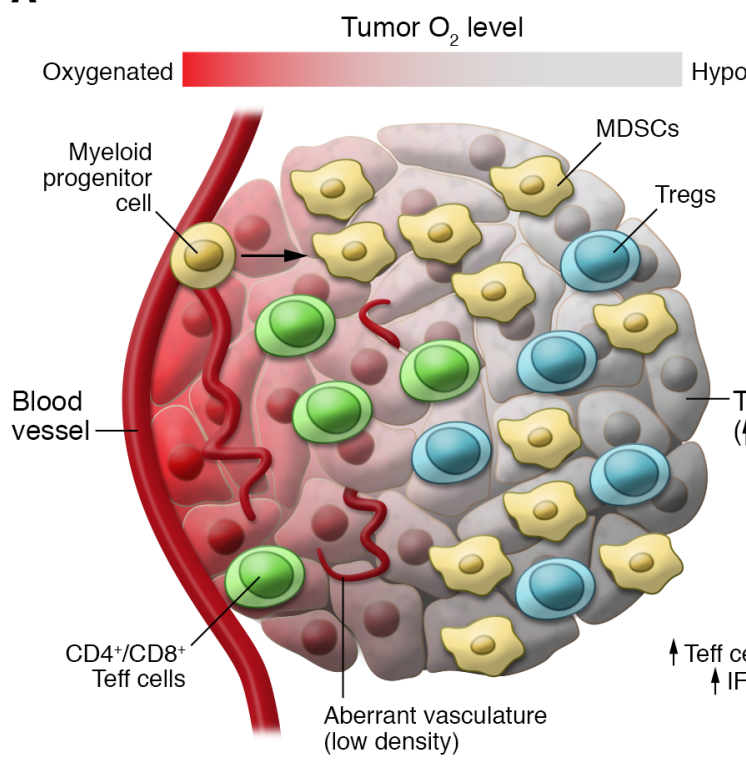

B Hypoxic

Figure 1. Combined therapy targeting hypoxia and immune checkpoints reduces immunosuppression within hypoxic zones in TRAMP prostate cancer. (A) Untreated TRAMP-C2 tumors exhibit hypoxic zones infiltrated by myeloid-derived suppressor cells (MDSCs) and Tregs, but few T effector (Teff) cells. Vascularization is at low density and aberrant. Suppressive MDSCs can be generated from myeloid progenitor cells. (B) Treatment with the hypoxia-activated prodrug TH-302 combined with anti-CTLA-4/anti-PD-1-blocking antibodies reduces hypoxia and changes the immune infiltrate in residual hypoxic areas. Vessel density increases and vessels display normalized morphology. Fewer arginase-expressing MDSCs can be seen. Generation of these suppressive MDSCs from myeloid progenitor cells is reduced. CD4 $4^{+}$and $C D 8^{+}$Teff cells infiltrate all areas of the tumor, proliferate, express effector cytokines and granzyme B, and show reduced active caspase-3 expression as a marker of apoptosis. These features are associated with enhanced survival.

\section{HAPs and immunotherapy in prostate cancer models}

In this issue of the JCI, Jayaprakash et al. investigated whether the use of an HAP could improve or reveal ICB responsiveness in prostate cancer models (13). The rationale is based on several observations of human prostate cancer, and the clinical need for new therapies for currently incurable metastatic castration-resistant prostate cancer (mCRPC). Indeed, mCRPC immunotherapy (using the sipuleucel-T cancer vaccine) has offered some modest clinical benefit for some patients, validating the potential for immune control of this malignancy. However, other therapeutic vaccines were unsuccessful in phase III trials, and recent ICB therapy was largely disappointing (14). Although there are numerous possibilities to account for therapy resistance, the current study focused on the hypoxic nature of the TME, a feature of aggressive human prostate cancer (15) and recapitulated in the transgenic adenocarcinoma of the mouse prostate (TRAMP) models used (13). Jayaprakash et al. observed that although TRAMP tumors were infiltrated by $\mathrm{CD} 4^{+}$and $\mathrm{CD} 8^{+} \mathrm{T}$ cells, they were generally excluded from hypox- ic regions. They hypothesized that targeting hypoxia concomitantly with ICB would extend the antitumor activity of reinvigorated $\mathrm{T}$ cells to these tumor sanctuaries. TH-302, a class II HAP that releases the active cytotoxic drug bromo-isophosphamide upon reduction in a hypoxic environment, was employed. As monotherapy, TH-302 was moderately efficacious in prolonging survival of mice implanted subcutaneously with TRAMP-C2 cells, but in combination with anti-CTLA-4/anti-PD-1 ICB, more than $80 \%$ of mice survived long-term (14). It should be noted that mice implanted with TRAMP-C2 were relatively responsive to ICB alone, with more than $50 \%$ long-term survival after ICB monotherapy. However, in the more challenging TRAMP transgenic mouse model in which ICB was ineffective, the benefits of the TH-302 and ICB combined therapy were also encouraging, based on increased prostate weight - a surrogate readout for tumor growth - and progression of tumors from adenocarcinomas to more aggressive neuroendocrine tumors.

Survival benefit of therapies tested in mouse cancer models is rarely fully predictive for human cancer, whereas conserved mechanisms, exemplified by the success of ICB development, can be extremely informative. In this regard, the mechanistic data from Jayaprakash et al. makes a major contribution (13). TH-302 treatment was remarkably efficacious at decreasing hypoxic zones in the TRAMP-C2 tumors. Moreover, when combined with ICB, there was increased $\mathrm{T}$ cell infiltration, which the investigators suggest was facilitated by improved vascularization. Regarding $\mathrm{T}$ cell effector functions (granzyme $\mathrm{B}$ and cytokine expression), these were improved in mice treated with TH-302 and ICB combined therapy, as were $\mathrm{T}$ cell activation, proliferation, and lower $\mathrm{T}$ cell apoptosis in hypoxic zones. Enhanced $\mathrm{CD}^{+} \mathrm{T}$ cell functionality was correlated with reduced myeloid-derived suppressor cell (MDSC) proportions. These cells were suppressive in in vitro tests, expressed arginase, and were of the granulocytic subset. In addition, mice treated with $\mathrm{TH}-302$ and ICB combined therapy had reduced capacity to polarize adoptively transferred myeloid precursors to MDSCs.

Based on these findings that correlate with therapeutic efficacy, what could be the mode of action of TH-302 HAP in the 
TRAMP-C2 model? Since the cytotoxic drug is only released in zones of deep hypoxia, it should act mainly on proliferating tumor cells and MDSCs, sparing $\mathrm{T}$ cells because they are excluded from these areas. This may lead to tumorantigen release, activating a de novo $\mathrm{T}$ cell response, the quality and quantity of which could be enhanced by anti-CTLA4 in secondary lymphoid tissue, and antiPD-1 at the tumor site. At the same time, reduced MDSC activity could be central to improved accessibility of the tumor to $\mathrm{T}$ cell infiltration via vascular changes and reduced immunosuppression (Figure 1).

\section{Limitations of the study and perspectives for clinical translation}

The outcome of TH-302 and ICB combined therapy in the mouse models in the Jayaprakash et al. study generates optimism for similar approaches in human prostate cancer. However, the detailed mechanistic studies were mostly made in the ectopically implanted TRAMP-C2 model rather than the spontaneous TRAMP model, understandingly, because of the practicalities of analysis. Nevertheless, the spontaneous TRAMP mice are most likely a better model for human cancer. Immune cell parameters after efficacious TH-302 and ICB combined therapy of TRAMP mice were less conclusive than in the ectopic model, with changes noted in the $\mathrm{T}$ effector to Treg ratios, and in the numbers of monocytic MDSCs (13). It is therefore possible that dominant shapers of the TME in the spontaneous TRAMP model, and potentially in patients, differ from the TRAMP-C2 model. Besides the immune infiltration, other key prognostic indicators for ICB response are mutational load, as a generator of targetable neo- epitopes, and PD-L1 expression in the TME (16). Whether these parameters are modulated in the HAP-treated prostate cancer models used here (13) or whether they might contribute to successful combination therapy in future clinical studies will be important to determine. Oxygen consumption and deprivation in solid tumors is a dynamic process, which will rapidly change according to tumor progression and consequences of treatment. If interferon- $\gamma$-secreting $\mathrm{T}$ cells can be driven to infiltrate the tumor, vessel perfusion can be improved and hypoxia can be reduced even in the absence of direct hypoxia targeting (17). In the long-term assessment of human cancer immunotherapies, prioritization of direct hypoxia targeting will likely depend not only on global assessment of the TME, but also upon fine analyses of even small hypoxic islands to determine whether they serve as important reservoirs of untargeted tumor cells facilitating immune escape.

\section{Acknowledgments}

This work was supported by a grant from the MEDIC Foundation. I would like to thank members of the Walker and Dietrich labs for helpful discussions.

Address correspondence to: Paul R. Walker, Centre for Translational Research in Onco-Hematology, Division of Oncology, University Medical Centre (CMU), University of Geneva, 1 rue Michel-Servet, 1211 Geneva 4, Switzerland. Phone: 41.22.379.5079; Email:paul.walker@unige.ch.

1. Sharma P, Hu-Lieskovan S, Wargo JA, Ribas A. Primary, adaptive, and acquired resistance to cancer immunotherapy. Cell. 2017;168(4):707-723.

2. Zappasodi R, Merghoub T, Wolchok JD. Emerging concepts for immune checkpoint blockadebased combination therapies. Cancer Cell.
2018;33(4):581-598

3. Wilson WR, Hay MP. Targeting hypoxia in cancer therapy. Nat Rev Cancer. 2011;11(6):393-410.

4. Chouaib S, Noman MZ, Kosmatopoulos K, Curran MA. Hypoxic stress: obstacles and opportunities for innovative immunotherapy of cancer. Oncogene. 2017;36(4):439-445.

5. Vuillefroy de Silly R, Dietrich PY, Walker PR. Hypoxia and antitumor $\mathrm{CD} 8^{+} \mathrm{T}$ cells: an incompatible alliance? Oncoimmunology. 2016;5(12):e1232236.

6. Semenza GL. Hypoxia-inducible factors in physiology and medicine. Cell. 2012;148(3):399-408.

7. Palazon A, Goldrath AW, Nizet V, Johnson RS. HIF transcription factors, inflammation, and immunity. Immunity. 2014;41(4):518-528.

8. Hatfield SM, et al. Immunological mechanisms of the antitumor effects of supplemental oxygenation. Sci Transl Med. 2015;7(277):277ra30.

9. Scharping NE, Menk AV, Whetstone RD, Zeng X, Delgoffe GM. Efficacy of PD-1 blockade is potentiated by metformin-induced reduction of tumor hypoxia. Cancer Immunol Res. 2017;5(1):9-16

10. Cha JH, et al. Metformin promotes antitumor immunity via endoplasmic-reticulum-associated degradation of PD-L1. Mol Cell. 2018;71(4):606-620.e7

11. Baran N, Konopleva M. Molecular pathways: hypoxia-activated prodrugs in cancer therapy. Clin Cancer Res. 2017;23(10):2382-2390.

12. Caldwell CC, et al. Differential effects of physiologically relevant hypoxic conditions on $\mathrm{T}$ lymphocyte development and effector functions. JImmunol. 2001;167(11):6140-6149.

13. Jayaprakash P, et al. Targeted hypoxia reduction restores $\mathrm{T}$ cell infiltration and sensitizes prostate cancer to immunotherapy. J Clin Invest. 2018;128(11):5137-5149.

14. Comiskey MC, Dallos MC, Drake CG. Immunotherapy in prostate cancer: teaching an old dog new tricks. Curr Oncol Rep. 2018;20(9):75.

15. Hompland T, et al. Combined MR imaging of oxygen consumption and supply reveals tumor hypoxia and aggressiveness in prostate cancer patients. Cancer Res. 2018;78(16):4774-4785.

16. Topalian SL, Taube JM, Anders RA, Pardoll DM. Mechanism-driven biomarkers to guide immune checkpoint blockade in cancer therapy. Nat Rev Cancer. 2016;16(5):275-287.

17. Tian L, et al. Mutual regulation of tumour vesse normalization and immunostimulatory reprogramming. Nature. 2017;544(7649):250-254. 